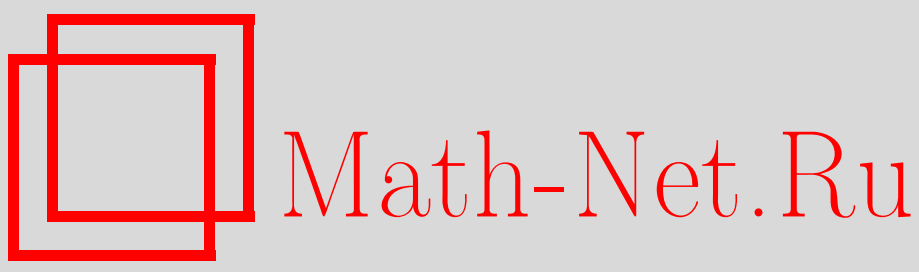

S. V. Agapov, On first integrals of two-dimensional geodesic flows, Sibirsk. Mat. Zh., 2020, Volume 61, Number 4, 721-734

DOI: https://doi.org/10.33048/smzh.2020.61.401

Use of the all-Russian mathematical portal Math-Net.Ru implies that you have read and agreed to these terms of use http://www . mathnet.ru/eng/agreement

Download details:

IP: 18.209 .158 .208

April 26, 2023, 08:55:25

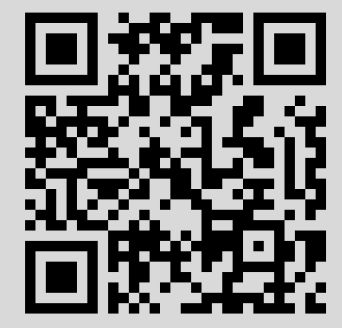


Сибирский математический журнал Июль-август, 2020. Том 61, № 4

УДК 517.938

\title{
О ПЕРВЫХ ИНТЕГРАЛАХ ДВУМЕРНЫХ ГЕОДЕЗИЧЕСКИХ ПОТОКОВ
}

\author{
C. В. Агапов
}

\begin{abstract}
Аннотация. Изучаются полиномиальные и рациональные по импульсам первые интегралы геодезических потоков (в том числе в магнитном поле) на двумерных поверхностях.
\end{abstract}

DOI 10.33048/smzh.2020.61.401

Ключевые слова: геодезический поток, первый интеграл, магнитное поле.

\section{1. Введение}

Геодезический поток римановой метрики $d s^{2}=g_{i j} d x^{i} d x^{j}$ на двумерной поверхности задается гамильтоновой системой

$$
\dot{x}^{j}=\left\{x^{j}, H\right\}, \quad \dot{p}_{j}=\left\{p_{j}, H\right\}, \quad H=\frac{1}{2} g^{i j} p_{i} p_{j}, \quad i, j=1,2,
$$

скобка Пуассона имеет вид

$$
\{F, H\}=\sum_{i=1}^{2}\left(\frac{\partial F}{\partial x^{i}} \frac{\partial H}{\partial p_{i}}-\frac{\partial F}{\partial p_{i}} \frac{\partial H}{\partial x^{i}}\right) .
$$

Геодезический поток (1.1) называется вполне интегрируемым, если существует дополнительный первый интеграл, т. е. такая функция $F(x, p)$, что $\frac{d F}{d t}=$ $\{F, H\} \equiv 0$, при этом $F$ и $H$ функционально независимы почти всюду.

Вопрос о том, какие метрики существуют на двумерных поверхностях с интегрируемым геодезическим потоком, классический. Известно, что на поверхностях рода больше 1 не существует аналитических римановых метрик, геодезический поток которых был бы вполне интегрируем в классе аналитических первых интегралов [1]. В гладком случае этот результат, вообще говоря, неверен: для любой гладкой поверхности можно указать такой гамильтониан $H$, что соответствующая гамильтонова система будет обладать бесконечно дифференцируемым интегралом, независимым (точнее, не всюду зависимым) с гамильтонианом. Подробное обсуждение этого вопроса можно найти в [2, гл. 3].

В подавляющем большинстве известных примеров первые интегралы являются полиномами по импульсам (или функциями от полиномов). Список метрик на двумерных сфере и торе, геодезический поток которых обладает дополнительным первым интегралом, линейным или квадратичным по импульсам,

Разд. 2, 4 написаны при финансовой поддержке Лаборатории топологии и динамики Новосибирского государственного университета (грант правительства РФ № 14.Ү26.31.0025). Разд. 3 написан при финансовой поддержке гранта РФФИ «Нелинейные системы и геометрия» № 18-01-00411.

(c) 2020 Агапов С. B. 
можно найти в [3]. На двумерной сфере также известны интегрируемые примеры геодезических потоков с первым интегралом третьей или четвертой степени (см., например, [4]). Существуют ли на этих поверхностях аналитические римановы метрики, геодезический поток которых обладает неприводимыми полиномиальными интегралами более высоких степеней, неизвестно. Различные вопросы, связанные с интегрируемыми геодезическими потоками на двумерном торе, изучались во многих работах (см., например, [5-9]).

С другой стороны, первые интегралы можно искать, скажем, в классе мероморфных функций. По сравнению с полиномиальными интегралами рациональные интегралы механических систем в целом исследованы в несколько меньшей степени (см., например, [10-17]). В [18] доказано, что на двумерных поверхностях существуют аналитические римановы метрики, геодезический поток которых допускает (локально) неприводимые рациональные по импульсам первые интегралы с числителем и знаменателем сколь угодно высоких степеней. Отметим, однако, что явных примеров таких метрик известно крайне мало (см. [19-21]). Более того, мало что известно о том, могут ли эти метрики (и соответствующие интегралы) существовать во всем фазовом пространстве.

Магнитный геодезический поток метрики $d s^{2}=g_{i j} d x^{i} d x^{j}$ на двумерной поверхности задается гамильтоновой системой

$$
\dot{x}^{j}=\left\{x^{j}, H\right\}_{m g}, \quad \dot{p}_{j}=\left\{p_{j}, H\right\}_{m g}, \quad H=\frac{1}{2} g^{i j} p_{i} p_{j}, \quad i, j=1,2,
$$

магнитная скобка Пуассона имеет вид

$$
\{F, H\}_{m g}=\sum_{i=1}^{2}\left(\frac{\partial F}{\partial x^{i}} \frac{\partial H}{\partial p_{i}}-\frac{\partial F}{\partial p_{i}} \frac{\partial H}{\partial x^{i}}\right)+\Omega\left(x^{1}, x^{2}\right)\left(\frac{\partial F}{\partial p_{1}} \frac{\partial H}{\partial p_{2}}-\frac{\partial F}{\partial p_{2}} \frac{\partial H}{\partial p_{1}}\right)
$$

где $\omega=\Omega\left(x^{1}, x^{2}\right) d x^{1} \wedge d x^{2}$ - замкнутая 2-форма, которая задает магнитное поле.

Напомним некоторые результаты об интегрируемых магнитных геодезических потоках на двумерном торе. Известны следующие интегрируемые случаи (с дополнительным первым интегралом на всех уровнях энергии).

Пример 1. Пусть метрика и магнитное поле имеют вид

$$
d s^{2}=d x^{2}+d y^{2}, \quad \omega=B d x \wedge d y, \quad B=\text { const } \neq 0 .
$$

Тогда магнитный геодезический поток (1.2) вполне интегрируем и существует первый интеграл вида

$$
F_{0}=\cos \left(\frac{p_{1}}{B}-y\right)
$$

Любопытно отметить, что ввиду результатов из [22] магнитный геодезический поток на двумерном торе в примере 1 не допускает полиномиальных по импульсам первых интегралов, не зависящих от интеграла энергии.

Пример 2. Пусть метрика и магнитное поле имеют вид

$$
d s^{2}=\Lambda(y)\left(d x^{2}+d y^{2}\right), \quad \omega=-u^{\prime}(y) d x \wedge d y .
$$

Тогда магнитный геодезический поток (1.2) вполне интегрируем и существует линейный по импульсам первый интеграл вида

$$
F_{1}=p_{1}+u(y)
$$


Отметим, что в присутствии магнитного поля интегрируемость геодезического потока на одном или на нескольких уровнях энергии, вообще говоря, не влечет интегрируемости одновременно на всех уровнях (в отличие от стандартного геодезического потока в нулевом магнитном поле). В [23] установлено, что квадратичный первый интеграл потока (1.2) на двумерном торе на двух различных уровнях энергии всегда сводится к линейному интегралу $F_{1}$ на всех уровнях. В [24] доказано, что если геодезический поток (1.2) в ненулевом магнитном поле обладает полиномиальным первым интегралом произвольной степени $N$ на $N+2$ различных уровнях энергии, то этот же интеграл сохраняется и на всех уровнях, при этом обязательно существует линейный по импульсам первый интеграл также на всех уровнях энергии.

С другой стороны, при фиксированном уровне энергии на двумерном торе существуют интегрируемые примеры магнитных геодезических потоков, отличные от примеров 1,2 . В $[25,26]$ построены примеры квадратичного по импульсам первого интеграла, не зависящего от гамильтониана. Вопрос о существовании интегралов степени выше 2 на одном уровне энергии, насколько нам известно, открыт. Различные результаты о магнитных геодезических потоках можно найти во многих работах (см., например, [27-34]).

Данная работа устроена следующим образом. В разд. 2 исследуются рациональные по импульсам первые интегралы геодезических потоков в случае, когда пространством положений является двумерный тор. В разд. 3 изучается вопрос о локальном существовании таких интегралов, приведены некоторые известные примеры, а также построен пример суперинтегрируемой гамильтоновой системы. Разд. 4 посвящен исследованию геодезических потоков в присутствии магнитного поля. Улучшен результат о полиномиальной интегрируемости таких потоков на двумерном торе, полученный ранее в [24]. Кроме того, в разд. 4 приведены некоторые результаты о рациональных первых интегралах магнитных геодезических потоков.

\section{2. Рациональные интегралы геодезических потоков на двумерном торе}

Далее будем работать в конформных координатах $d s^{2}=\Lambda(x, y)\left(d x^{2}+d y^{2}\right)$. Гамильтониан принимает вид $H=\frac{p_{1}^{2}+p_{2}^{2}}{2 \Lambda(x, y)}$.

Покажем, что если геодезический поток на двумерной поверхности обладает рациональным по импульсам первым интегралом, то коэффициенты этого интеграла удовлетворяют соотношению специального вида. А именно, имеет место

Лемма 1. Предположим, что геодезический поток метрики

$$
d s^{2}=\Lambda(x, y)\left(d x^{2}+d y^{2}\right)
$$

на двумерной поверхности обладает рациональным по импульсам первым интегралом

$$
F=\frac{\sum_{k=0}^{M} a_{k}(x, y) p_{1}^{M-k} p_{2}^{k}}{\sum_{s=0}^{N} b_{s}(x, y) p_{1}^{N-s} p_{2}^{s}}
$$

$c$ аналитическими коэффициентами $a_{k}(x, y), b_{s}(x, y)$. Положим

$$
f_{1}=a_{0}-a_{2}+a_{4}-\ldots, \quad g_{1}=a_{1}-a_{3}+a_{5}-\ldots,
$$




$$
f_{2}=b_{0}-b_{2}+b_{4}-\ldots, \quad g_{2}=b_{1}-b_{3}+b_{5}-\ldots,
$$

здесь $a_{k}=0$ при $k>M, b_{s}=0$ при $s>N$. Тогда имеет место соотношение

$$
\left(\frac{f_{1}-i g_{1}}{f_{2}-i g_{2}}\right)_{x}-i\left(\frac{f_{1}-i g_{1}}{f_{2}-i g_{2}}\right)_{y}=0
$$

Отметим, что для полиномиальных первых интегралов аналогичное соотношение хорошо известно, оно получено В. Н. Колокольцовым в [3].

ДоКАЗАТЕЛЬСТво. Положим

$$
Q_{M}=\sum_{k=0}^{M} a_{k}(x, y) p_{1}^{M-k} p_{2}^{k}, \quad R_{N}=\sum_{s=0}^{N} b_{s}(x, y) p_{1}^{N-s} p_{2}^{s} .
$$

Тогда $F=\frac{Q_{M}}{R_{N}}$. Из равенства $2 \Lambda^{2} R_{N}^{2}\{F, H\}=0$ получим

$$
\begin{aligned}
& 2 \Lambda p_{1}\left(\left(Q_{M}\right)_{x} R_{N}-Q_{M}\left(R_{N}\right)_{x}\right)+2 \Lambda p_{2}\left(\left(Q_{M}\right)_{y} R_{N}-Q_{M}\left(R_{N}\right)_{y}\right) \\
+ & \Lambda_{x}\left(p_{1}^{2}+p_{2}^{2}\right)\left(\left(Q_{M}\right)_{p_{1}} R_{N}-Q_{M}\left(R_{N}\right)_{p_{1}}\right)+\Lambda_{y}\left(p_{1}^{2}+p_{2}^{2}\right)\left(\left(Q_{M}\right)_{p_{2}} R_{N}-Q_{M}\left(R_{N}\right)_{p_{2}}\right)=0 .
\end{aligned}
$$

Это однородный полином по импульсам степени $M+N+1$. Равенство нулю коэффициентов при мономах $p_{1}^{M+N+1-l} p_{2}^{l}$ дает следующую систему уравнений:

$$
\begin{aligned}
& S_{l} \equiv \sum_{k=0}^{M}\left\{\Lambda_{x} a_{k}(\right.\left.b_{l-k}(M-N+l-2 k)+b_{l-k-2}(M-N+l-2 k-2)\right) \\
&+\Lambda_{y} a_{k}\left(b_{l-k+1}(2 k-l-1)+b_{l-k-1}(2 k-l+1)\right) \\
&\left.+2 \Lambda\left(a_{k x} b_{l-k}-a_{k} b_{l-k_{x}}+a_{k y} b_{l-k-1}-a_{k} b_{l-k-1}\right)\right\}=0,
\end{aligned}
$$

здесь $l=0,1, \ldots, M+N+1$, причем $a_{k}=0$ при $k<0, k>M$ и $b_{s}=0$ при $s<0, s>N$. Положим

$$
U=\frac{1}{2 \Lambda}\left(S_{0}-S_{2}+S_{4}-\ldots\right), \quad V=\frac{1}{2 \Lambda}\left(S_{1}-S_{3}+S_{5}-\ldots\right) .
$$

Поскольку $S_{l}=0$ для всех $l$, получаем $U=V=0$. Прямыми вычислениями нетрудно убедиться, что равенство $U-i V=0$ эквивалентно соотношению

$$
\left(\frac{f_{1}-i g_{1}}{f_{2}-i g_{2}}\right)_{x}-i\left(\frac{f_{1}-i g_{1}}{f_{2}-i g_{2}}\right)_{y}=0
$$

что и требовалось. Лемма 1 доказана.

Поскольку на двумерном торе конформные координаты можно ввести глобально, можно воспользоваться предыдущей леммой, чтобы доказать следующее утверждение.

Теорема 1. Предположим, что геодезический поток метрики

$$
d s^{2}=\Lambda(x, y)\left(d x^{2}+d y^{2}\right)
$$

на двумерном торе обладает рациональным по импульсам первым интегралом

$$
F=\frac{a_{0}(x, y) p_{1}+a_{1}(x, y) p_{2}}{b_{0}(x, y) p_{1}+b_{1}(x, y) p_{2}}
$$


Предположим, что все коэффициенты $a_{k}(x, y), b_{s}(x, y)$ - аналитические функции, периодические по обоим аргументам, причем хотя бы один из этих коэффициентов нигде не обращается в нуль. Тогда конформный множитель $\Lambda(x, y)$ постоянен, т. е. не зависит от координат.

ДокАзАтЕльство. Пусть, например, $b_{0}(x, y)$ нигде не обращается в нуль. По лемме 1 имеем

$$
\left(\frac{a_{0}-i a_{1}}{b_{0}-i b_{1}}\right)_{x}-i\left(\frac{a_{0}-i a_{1}}{b_{0}-i b_{1}}\right)_{y}=0 .
$$

Перепишем это равенство в виде

$$
(u+i v)_{x}-i(u+i v)_{y}=0,
$$

где

$$
u=\frac{a_{0} b_{0}+a_{1} b_{1}}{b_{0}^{2}+b_{1}^{2}}, \quad v=\frac{a_{0} b_{1}-a_{1} b_{0}}{b_{0}^{2}+b_{1}^{2}} .
$$

Следовательно, $u_{x x}+u_{y y}=v_{x x}+v_{y y}=0$, т. е. функции $u, v$ гармонические. Ввиду их периодичности получаем, что $u(x, y) \equiv C_{1}, v(x, y) \equiv C_{2}$, где $C_{1}, C_{2}-$ некоторые постоянные. Из этих равенств выразим $a_{0}(x, y), a_{1}(x, y)$ :

$$
a_{0}=C_{1} b_{0}+C_{2} b_{1}, \quad a_{1}=-C_{2} b_{0}+C_{1} b_{1} .
$$

Заметим, что если $v(x, y) \equiv C_{2}=0$, то из равенства $a_{0} b_{1}-a_{1} b_{0}=0$ следует, что первый интеграл $F$ вообще не зависит от импульсов. Поэтому далее считаем, что $C_{2} \neq 0$.

Равенство нулю скобки Пуассона дает еще два уравнения $S_{0}=0, S_{1}=0$ (см. доказательство леммы 1 при $M=N=1$ ):

$$
\begin{aligned}
& \Lambda_{x}\left(b_{0}^{2}+b_{1}^{2}\right)+2 \Lambda\left(b_{0} b_{1 y}-b_{1} b_{0 y}\right)=0, \\
& \Lambda_{y}\left(b_{0}^{2}+b_{1}^{2}\right)+2 \Lambda\left(b_{1} b_{0 x}-b_{0} b_{1 x}\right)=0 .
\end{aligned}
$$

Пусть $\Lambda(x, y)=e^{w(x, y)}, b_{1}(x, y)=b_{0}(x, y) g(x, y)$. Уравнения $(2.2),(2.3)$ примут вид

$$
\left(1+g^{2}\right) w_{y}=2 g_{x}, \quad\left(1+g^{2}\right) w_{x}=-2 g_{y} .
$$

Отсюда

$$
w_{x} g_{x}+w_{y} g_{y}=0 .
$$

Продифференцируем первое уравнение (2.4) по $y$, второе по $x$ и результаты сложим. В силу (2.5) получим $w_{x x}+w_{y y}=0$. Ввиду периодичности всех функций отсюда следует, что функция $w(x, y)$ и, следовательно, метрика $\Lambda(x, y)$ не зависят от координат. Теорема 1 доказана.

\section{3. Локальные интегралы}

Из доказательства теоремы 1 видно, что глобальное существование рационального интеграла на двумерном торе накладывает ограничения на коэффициенты интеграла, в частности, возникающие в доказательстве гармонические функции должны быть постоянны. Посмотрим, что произойдет, если отбросим это требование и попробуем построить хотя бы локальные интегрируемые примеры. 
Пусть геодезический поток метрики $d s^{2}=\Lambda(x, y)\left(d x^{2}+d y^{2}\right)$ на двумерной поверхности обладает локальным рациональным по импульсам первым интегралом

$$
F=\frac{a_{0}(x, y) p_{1}+a_{1}(x, y) p_{2}}{b_{0}(x, y) p_{1}+b_{1}(x, y) p_{2}}
$$

Поскольку исследование общего случая представляет собой непростую задачу, в данной работе ограничимся разбором самого простого случая, когда коэффициенты этого интеграла удовлетворяют соотношениям (2.1). Будем придерживаться тех же обозначений, что и при доказательстве теоремы 1:

$$
\Lambda(x, y)=e^{w(x, y)}, \quad b_{1}(x, y)=b_{0}(x, y) g(x, y) .
$$

Из уравнений (2.4) выразив $w_{x}, w_{y}$ и перекрестным дифференцированием избавившись от них, получим уравнение на функцию $g(x, y)$ :

$$
\left(\frac{g_{x}}{g^{2}+1}\right)_{x}+\left(\frac{g_{y}}{g^{2}+1}\right)_{y}=0
$$

или, что то же самое,

$$
(\operatorname{arctg} g)_{x x}+(\operatorname{arctg} g)_{y y}=0 .
$$

Положим $g(x, y)=\operatorname{tg} z(x, y)$, где $z(x, y)$ - произвольная гармоническая функция. Из уравнений (2.4) получим

$$
w_{x}=-2 z_{y}, \quad w_{y}=2 z_{x},
$$

откуда, в частности, следует, что $w(x, y)$ тоже гармоническая функция. Итак, геодезический поток метрики $\Lambda(x, y)=e^{w(x, y)}$ обладает первым интегралом $\widetilde{F}=\frac{F-C_{1}}{C_{2}}$ вида

$$
\widetilde{F}=\frac{p_{1} \sin z-p_{2} \cos z}{p_{1} \cos z+p_{2} \sin z} .
$$

Оказывается, однако, что при этом существуют сразу два линейных интеграла $f_{1}, f_{2}$ :

$$
f_{1}=\left(p_{1} \sin z-p_{2} \cos z\right) e^{-\frac{w}{2}}, \quad f_{2}=\left(p_{1} \cos z+p_{2} \sin z\right) e^{-\frac{w}{2}},
$$

в чем легко убедиться непосредственной проверкой. При этом

$$
f_{1}^{2}+f_{2}^{2}=2 H, \quad \frac{f_{2}}{f_{1}}=\widetilde{F} .
$$

Наличие двух линейных интегралов характерно для геодезических потоков плоских метрик. Действительно, гауссова кривизна построенной метрики равняется нулю: $K=-\frac{1}{2} e^{-w}\left(w_{x x}+w_{y y}\right) \equiv 0$.

Зафиксируем некоторые значения первых интегралов: $f_{1}=c_{1}, f_{2}=c_{2}$. Уравнения геодезических принимают вид

$$
\frac{d x}{d t}= \pm \frac{\left(c_{1} \sin z+c_{2} \cos z\right)}{e^{w / 2}}, \quad \frac{d y}{d t}= \pm \frac{\left(c_{2} \sin z-c_{1} \cos z\right)}{e^{w / 2}} .
$$

Полагая $y=y(x)$ вдоль геодезической, приходим к одному уравнению:

$$
\frac{d y}{d x}=\frac{c_{2} \sin z-c_{1} \cos z}{c_{1} \sin z+c_{2} \cos z}
$$

Итак, имеет место 
Предложение 1. Предположим, что $w(x, y)$ - произвольная гармоническая функция, т. е. $w$ удовлетворяет уравнению $w_{x x}+w_{y y}=0$. Тогда метрика $d s^{2}=e^{w(x, y)}\left(d x^{2}+d y^{2}\right)$ плоская, а ее геодезический поток обладает двумя линейными по импульсам первыми интегралами

$$
f_{1}=\left(p_{1} \sin z-p_{2} \cos z\right) e^{-\frac{w}{2}}, \quad f_{2}=\left(p_{1} \cos z+p_{2} \sin z\right) e^{-\frac{w}{2}},
$$

где $z(x, y)$ - гармоническая функция, связанная с $w$ соотношениями $w_{x}=-2 z_{y}$, $w_{y}=2 z_{x}$. При фиксированных значениях первых интегралов $\left\{f_{1}=c_{1}, f_{2}=c_{2}\right\}$ геодезические удовлетворяют уравнению

$$
\frac{d y}{d x}=\frac{c_{2} \sin z-c_{1} \cos z}{c_{1} \sin z+c_{2} \cos z} .
$$

Ввиду наличия достаточного числа первых интегралов это уравнение интегрируется в квадратурах.

Приведем несколько простых примеров.

Положим $w(x, y)=\log \left(x^{2}+y^{2}\right)$. Тогда $z(x, y)=\operatorname{arctg}\left(-\frac{y}{x}\right)$. Геодезические удовлетворяют уравнению $\frac{d y}{d x}=\frac{c_{1} x+c_{2} y}{c_{1} y-c_{2} x}$. После замены $y(x)=x t(x)$ переменные разделяются:

$$
\int \frac{d x}{x}=\int \frac{\left(c_{1} t-c_{2}\right) d t}{c_{1}\left(1-t^{2}\right)+2 c_{2} t}+\tilde{c} .
$$

Интеграл в правой части легко берется. После возвращения к исходным переменным в итоге получаем

ПримеР 3. Геодезические метрики $d s^{2}=\left(x^{2}+y^{2}\right)\left(d x^{2}+d y^{2}\right)$ задаются уравнением

$$
c_{1}\left(x^{2}-y^{2}\right)+2 c_{2} x y=c_{0}^{2}, \quad c_{0}, c_{1}, c_{2} \in \mathbb{R} .
$$

При $c_{0}=0$ это две прямые, пересекающиеся в начале координат, при $c_{0} \neq 0-$ гиперболы.

Положим $w(x, y)=-\log \left(x^{2}+y^{2}\right)$. Тогда $z(x, y)=\operatorname{arctg}\left(\frac{y}{x}\right)$. Аналогичными рассуждениями получим

ПримеР 4. Геодезические метрики $d s^{2}=\frac{1}{x^{2}+y^{2}}\left(d x^{2}+d y^{2}\right)$ задаются уравнением

$$
\left(x^{2}+y^{2}\right) e^{\frac{2 c_{2}}{c_{1}} \operatorname{arctg} \frac{y}{x}}=c_{0}^{2}, \quad c_{0}, c_{1}, c_{2} \in \mathbb{R} .
$$

В полярной системе координат $(\rho, \varphi)$ это уравнение принимает вид

$$
\rho=\left|c_{0}\right| e^{-\frac{c_{2}}{c_{1}} \varphi} .
$$

Для полноты изложения приведем некоторые известные примеры рациональных интегралов.

1. В [16] найдены две функции

$$
H=\frac{p_{1}^{2}+p_{2}^{2}}{2}+f\left(p_{1}, p_{2}\right)\left(x p_{1}-\alpha y p_{2}\right), \quad F=p_{1}^{\alpha} p_{2}, \quad \alpha \in \mathbb{R},
$$

коммутирующие относительно стандартной скобки Пуассона. Здесь $f\left(p_{1}, p_{2}\right)-$ произвольная функция импульсов $p_{1}, p_{2}$. В зависимости от параметра $\alpha$ первый интеграл $F$ может быть полиномиальным, рациональным, алгебраическим или даже трансцендентным. Как отмечено в [19], если положить $f\left(p_{1}, p_{2}\right)=$ 
$p_{1}+p_{2}, \alpha=-\frac{s}{r}$, где $r, s-$ взаимно простые натуральные числа, то получим рациональный по импульсам первый интеграл геодезического потока:

$$
H=\left(x+\frac{1}{2}\right) p_{1}^{2}+\left(x+\frac{s}{r} y\right) p_{1} p_{2}+\left(\frac{1}{2}+\frac{s}{r} y\right) p_{2}^{2}, \quad F_{0}=F^{r}=\frac{p_{2}^{r}}{p_{1}^{s}} .
$$

С другой стороны, можно положить $f\left(p_{1}, p_{2}\right) \equiv c$, где $c$ - произвольная постоянная. Тогда $2 H=\left(p_{1}+c x\right)^{2}+\left(p_{2}-\alpha c y\right)^{2}-c^{2}\left(x^{2}+\alpha^{2} y^{2}\right), F=p_{1}^{\alpha} p_{2}$. Это позволяет построить пример натуральной механической системы с гамильтонианом

и первым интегралом

$$
\widetilde{H}=\frac{1}{2}\left(p_{1}^{2}+p_{2}^{2}\right)-\frac{c^{2}}{2}\left(x^{2}+\alpha^{2} y^{2}\right)
$$

$$
\widetilde{F}=\left(p_{1}-c x\right)^{\alpha}\left(p_{2}+\alpha c y\right), \quad\{\widetilde{H}, \widetilde{F}\}=0 .
$$

Построенная гамильтонова система суперинтегрируема. Действительно, при $\alpha= \pm n$ имеем

$$
\begin{gathered}
\widetilde{H}=\frac{p_{1}^{2}+p_{2}^{2}}{2}-\frac{c^{2}}{2}\left(x^{2}+n^{2} y^{2}\right), \\
\widetilde{F}_{n}=\left(p_{1}-c x\right)^{n}\left(p_{2}+n c y\right), \quad \widetilde{F}_{-n}=\frac{p_{2}-n c y}{\left(p_{1}-c x\right)^{n}}, \\
\left\{\widetilde{H}, \widetilde{F}_{n}\right\}=\left\{\widetilde{H}, \widetilde{F}_{-n}\right\}=0,
\end{gathered}
$$

и функции $\widetilde{H}, \widetilde{F}_{n}, \widetilde{F}_{-n}$ функционально независимы почти всюду. Заметим, что в этом случае имеются также квадратичные по импульсам интегралы

$$
\begin{gathered}
F_{1}=\widetilde{F}_{n} \widetilde{F}_{-n}=p_{2}^{2}-n^{2} c^{2} y^{2}, \quad F_{2}=2 \widetilde{H}-F_{1}=p_{1}^{2}-c^{2} x^{2}, \\
\left\{\widetilde{H}, F_{1}\right\}=\left\{\widetilde{H}, F_{2}\right\}=\left\{F_{1}, F_{2}\right\}=0 .
\end{gathered}
$$

2. В [20] найдены две функции

$$
H=\frac{1}{2} p_{1}^{2}+\frac{1}{2}\left(p_{2}-\frac{x}{y}\right)^{2}-\frac{1}{2}\left(\frac{x}{y}\right)^{2}, \quad F=\frac{x p_{2}-y p_{1}+y}{p_{2}},
$$

которые коммутируют относительно стандартной скобки Пуассона. Эти две функции порождают новые:

$$
\widetilde{H}=\frac{p_{1}^{2}+p_{2}^{2}}{2}-\frac{1}{2}\left(\frac{x}{y}\right)^{2}, \quad \widetilde{F}=\frac{p_{2} x y-p_{1} y^{2}+x^{2}+y^{2}}{x+p_{2} y}, \quad\{\widetilde{H}, \widetilde{F}\}_{m g}=0,
$$

что дает пример интегрируемой натуральной механической системы в магнитном поле $w=\frac{1}{y} d x \wedge d y$.

3. Упомянем здесь пример рационального интеграла геодезического потока, который был найден М. В. Павловым и С. П. Царевым. Пусть гамильтониан и первый интеграл имеют вид

$$
H=\frac{1}{2}\left(p_{1}^{2}+\frac{p_{2}^{2}}{\left(b_{1}-b_{2}\right)^{2}}\right), \quad F=\frac{\left(b_{1}-b_{2}\right) p_{1}-b_{2} p_{2}}{\left(b_{1}-b_{2}\right) p_{1}-b_{1} p_{2}},
$$

здесь $b_{j}=b_{j}(x, t), j=1,2$. Условие $\{F, H\}=0$ эквивалентно системе уравнений

$$
b_{1 t}=\left(1+b_{1} b_{2}\right) b_{1 x}-\left(1+b_{1}^{2}\right) b_{2 x}, \quad b_{2 t}=\left(1+b_{2}^{2}\right) b_{1 x}-\left(1+b_{1} b_{2}\right) b_{2 x} .
$$


Система (3.2) полугамильтонова (см. [35]). В новых переменных

$$
u(x, t)=-\frac{2}{\left(b_{1}-b_{2}\right)^{2}}, \quad v(x, t)=\frac{b_{1}+b_{2}}{b_{1}-b_{2}}
$$

она записывается в виде законов сохранения:

$$
u_{t}+2 v_{x}=0, \quad v_{t}+(\log u)_{x}=0 .
$$

Более того, в гиперболической области она обладает инвариантами Римана $r_{1}(x, t), r_{2}(x, t)$ и может быть записана в диагональной форме:

$$
r_{1 x}=\frac{1}{4}\left(r_{1}-r_{2}\right) r_{1 t}, \quad r_{2 x}=-\frac{1}{4}\left(r_{1}-r_{2}\right) r_{2 t},
$$

здесь $8 u=\left(r_{1}-r_{2}\right)^{2}, 2 v=-\left(r_{1}+r_{2}\right)$. Классический метод годографа (см., например, [36]) меняет местами независимые и зависимые переменные. Примененный к системе (3.3), он дает следующие соотношения:

$$
\frac{\partial t}{\partial r_{2}}=\frac{1}{4}\left(r_{1}-r_{2}\right) \frac{\partial x}{\partial r_{2}}, \quad \frac{\partial t}{\partial r_{1}}=-\frac{1}{4}\left(r_{1}-r_{2}\right) \frac{\partial x}{\partial r_{1}} .
$$

Перекрестным дифференцированием избавимся от функции $t\left(r_{1}, r_{2}\right)$, что приводит нас к уравнению Эйлера - Пуассона - Дарбу на $x\left(r_{1}, r_{2}\right)$ :

$$
\frac{\partial^{2} x}{\partial r_{1} \partial r_{2}}=\frac{1}{2\left(r_{1}-r_{2}\right)}\left(\frac{\partial x}{\partial r_{1}}-\frac{\partial x}{\partial r_{2}}\right) .
$$

По любому решению этого уравнения (см., например, работу [37] и ссылки в ней) с помощью соотношения (3.4) можно найти функцию $t$ в квадратурах. Обращая затем $x\left(r_{1}, r_{2}\right), t\left(r_{1}, r_{2}\right)$, можно восстановить метрику и коэффициенты первого интеграла (3.1).

\section{4. Магнитные геодезические потоки на двумерном торе}

Данный раздел посвящен исследованию геодезических потоков в магнитном поле. В [24] установлена связь между полиномиальной интегрируемостью магнитного геодезического потока на нескольких и на всех уровнях энергии. В данном разделе этот результат улучшен.

Лемма 2. Предположим, что магнитный геодезический поток (1.2) метрики $d s^{2}=\Lambda(x, y)\left(d x^{2}+d y^{2}\right)$ на двумерном торе обладает полиномиальным по импульсам первым интегралом $F$ произвольной степени $N$ на $\frac{N+1}{2}$ или $\frac{N+2}{2}$ (в зависимости от четности $N$ ) попарно различных уровнях энергии $\left\{H=C_{1}\right\},\{H=$ $\left.C_{2}\right\}, \ldots$ Здесь $C_{j}>0$ для любого $j$. Тогда $F$ является первым интегралом потока (1.2) одновременно на всех уровнях энергии.

ДокАЗАТЕльство. Пусть первый интеграл имеет вид

$$
F=\sum_{s=0}^{N} \sum_{k=0}^{s} a_{s, k}(x, y) p_{1}^{s-k} p_{2}^{k},
$$

где коэффициенты $a_{s, k}(x, y)$ - гладкие периодические функции обоих переменных. На фиксированном уровне энергии $H=\frac{p_{1}^{2}+p_{2}^{2}}{2 \Lambda(x, y)}=\frac{C}{2}$ параметризуем импульсы следующим образом: $p_{1}=\sqrt{C \Lambda} \cos \phi, p_{2}=\sqrt{C \Lambda} \sin \phi$. Первый интеграл 
(4.1) принимает вид

$$
\begin{aligned}
F=\sum_{s=0}^{N} \sum_{k=0}^{s}\left(\frac{C^{\frac{s}{2}}}{2^{s} i^{k}} a_{s, k}(x, y) \Lambda^{\frac{s}{2}}(x, y)\right) & \\
& \times\left(\sum_{l=0}^{s-k} \sum_{m=0}^{k}(-1)^{m}\left(\begin{array}{c}
s-k \\
l
\end{array}\right)\left(\begin{array}{c}
k \\
m
\end{array}\right) e^{i(s-2 l-2 m) \phi}\right) .
\end{aligned}
$$

Условие $\frac{d F}{d t}=0$ эквивалентно следующему соотношению (см. $\left.[28,24]\right)$ :

$$
F_{x} \cos \phi+F_{y} \sin \phi+F_{\phi}\left(\frac{\Lambda_{y}}{2 \Lambda} \cos \phi-\frac{\Lambda_{x}}{2 \Lambda} \sin \phi-\frac{\Omega}{\sqrt{C \Lambda}}\right)=0,
$$

которое перепишем в виде

$$
\begin{aligned}
e^{i \phi} \sqrt{C}\left(2 \Lambda F_{x}+\frac{2 \Lambda}{i} F_{y}\right. & \left.+\left(\Lambda_{y}-\frac{\Lambda_{x}}{i}\right) F_{\phi}\right)-4 \sqrt{\Lambda} \Omega F_{\phi} \\
& +e^{-i \phi} \sqrt{C}\left(2 \Lambda F_{x}-\frac{2 \Lambda}{i} F_{y}+\left(\Lambda_{y}+\frac{\Lambda_{x}}{i}\right) F_{\phi}\right)=0 .
\end{aligned}
$$

Подставим (4.2) в (4.3). Полученное соотношение имеет вид

$$
R_{1}+R_{0}+R_{-1}=0
$$

где

$$
\begin{aligned}
R_{1}= & \sum_{s=0}^{N} \sum_{k=0}^{s} \frac{C^{\frac{s+1}{2}}}{2^{s} i^{k}}\left\{\left(2 \Lambda\left(\Lambda^{\frac{s}{2}} a_{s, k}\right)_{x}+\frac{2 \Lambda}{i}\left(\Lambda^{\frac{s}{2}} a_{s, k}\right)_{y}\right)\right. \\
\times & \left(\sum_{l=0}^{s-k} \sum_{m=0}^{k}(-1)^{m}\left(\begin{array}{c}
s-k \\
l
\end{array}\right)\left(\begin{array}{c}
k \\
m
\end{array}\right) e^{i(s-2 l-2 m+1) \phi}\right)+i a_{s, k} \Lambda^{\frac{s}{2}}\left(\Lambda_{y}-\frac{\Lambda_{x}}{i}\right) \\
& \left.\times\left(\sum_{l=0}^{s-k} \sum_{m=0}^{k}(-1)^{m}\left(\begin{array}{c}
s-k \\
l
\end{array}\right)\left(\begin{array}{c}
k \\
m
\end{array}\right)(s-2 l-2 m) e^{i(s-2 l-2 m+1) \phi}\right)\right\}, \\
R_{0}= & \sum_{s=0}^{N} \sum_{k=0}^{s} \frac{C^{\frac{s}{2}}}{2^{s} i^{k}}\left\{\left(-4 \sqrt{\Lambda} \Omega i a_{s, k} \Lambda^{\frac{s}{2}}\right)\right. \\
\times & \left.\left(\sum_{l=0}^{s-k} \sum_{m=0}^{k}(-1)^{m}\left(\begin{array}{c}
s-k \\
l
\end{array}\right)\left(\begin{array}{c}
k \\
m
\end{array}\right)(s-2 l-2 m) e^{i(s-2 l-2 m) \phi}\right)\right\}, \\
R_{-1}= & \sum_{s=0}^{N} \sum_{k=0}^{s} \frac{C^{\frac{s+1}{2}}}{2^{s} i^{k}}\left\{\left(\begin{array}{c}
\left.2 \Lambda\left(\Lambda^{\frac{s}{2}} a_{s, k}\right)_{x}-\frac{2 \Lambda}{i}\left(\Lambda^{\frac{s}{2}} a_{s, k}\right)_{y}\right) \\
\times
\end{array}\right.\right. \\
& \left.\quad \sum_{l=0}^{s-k} \sum_{m=0}^{k}(-1)^{m}\left(\begin{array}{c}
s-k \\
l
\end{array}\right)\left(\begin{array}{c}
k \\
m
\end{array}\right) e^{i(s-2 l-2 m-1) \phi}\right)+i a_{s, k} \Lambda^{\frac{s}{2}}\left(\Lambda_{y}+\frac{\Lambda_{x}}{i}\right) \\
& \left.\times\left(\sum_{l=0}^{s-k} \sum_{m=0}^{k}(-1)^{m}\left(\begin{array}{c}
s-k \\
l
\end{array}\right)\left(\begin{array}{c}
k \\
m
\end{array}\right)(s-2 l-2 m) e^{i(s-2 l-2 m-1) \phi}\right)\right\} .
\end{aligned}
$$


Таким образом, левая часть (4.4) является полиномом по $e^{i \phi}$, следовательно, (4.4) имеет вид

$$
\sum_{n=-(N+1)}^{N+1} M_{n}(x, y) e^{i n \phi}=0,
$$

причем все $M_{n}(x, y)$ должны быть тождественно равны нулю. В частности, при $n=N+1$ после всех упрощений получим

$$
M_{N+1}(x, y)=\frac{(C \Lambda)^{\frac{N+1}{2}}}{2^{N-1}} \sum_{k=0}^{N}\left(\frac{\left(a_{N, k}\right)_{x}}{i^{k}}+\frac{\left(a_{N, k}\right)_{y}}{i^{k+1}}\right)=0 .
$$

Поскольку все коэффициенты первого интеграла $F$ вещественные, это дает два соотношения:

$$
\begin{gathered}
\left(a_{N, 0}-a_{N, 2}+a_{N, 4}-\ldots\right)_{x}=\left(a_{N, 1}-a_{N, 3}+a_{N, 5}-\ldots\right)_{y} \\
\left(a_{N, 0}-a_{N, 2}+a_{N, 4}-\ldots\right)_{y}=-\left(a_{N, 1}-a_{N, 3}+a_{N, 5}-\ldots\right)_{x} .
\end{gathered}
$$

Следовательно,

$$
a_{N, 0}-a_{N, 2}+a_{N, 4}-\ldots=K_{1}, \quad a_{N, 1}-a_{N, 3}+a_{N, 5}-\ldots=K_{2},
$$

где $K_{1}, K_{2}-$ константы Колокольцова.

В общем случае для произвольного $n$ выписать коэффициент $M_{n}(x, y)$ при $e^{i n \phi}$ в явном виде не так просто. Тем не менее заметим, что ввиду (4.4) $R_{1}$ дает вклад в $M_{n}$ лишь при $s-2 l-2 m+1=n$. Аналогичным образом в $R_{0}$ суммирование идет с учетом условия $s-2 l-2 m=n$ и, наконец, в $R_{-1}-$ с учетом условия $s-2 l-2 m-1=n$. Таким образом, если $n$ четное, то суммирование в $R_{1}, R_{-1}$ идет по нечетным $s: s=1,3,5, \ldots$, а в $R_{0}-$ по четным: $s=0,2,4, \ldots$. С другой стороны, в случае нечетного $n$ суммирование в $R_{1}, R_{-1}$ идет по четным $s: s=0,2,4, \ldots$, а в $R_{0}-$ по нечетным: $s=1,3,5, \ldots$. Примем также во внимание, что $\left.R_{0}\right|_{s=0}=0$. Предположим, что $N$ четное (случай нечетного $N$ разбирается аналогично). Тогда $M_{n}$ имеет вид

$$
M_{n}=C \sum_{j=0}^{\frac{N}{2}-1} \alpha_{j}(x, y) C^{j}=0
$$

в случае четного $n$ и

$$
M_{n}=\sqrt{C} \sum_{j=0}^{\frac{N}{2}} \alpha_{j}(x, y) C^{j}=0
$$

в случае нечетного $n$. Следовательно, если потребуем, чтобы $F$ был первым интегралом одновременно на $\frac{N}{2}+1$ уровне энергии, то все коэффициенты $\alpha_{j}(x, y)$ должны тождественно равняться нулю. Но это означает, что $F$ является интегралом одновременно на всех уровнях энергии. Лемма 2 доказана.

С учетом результатов, полученных в [24], из леммы 2 очевидным образом вытекает

Теорема 2. Предположим, что геодезический поток (1.2) аналитической римановой метрики $d s^{2}=\Lambda(x, y)\left(d x^{2}+d y^{2}\right)$ на двумерном торе в ненулевом магнитном поле обладает полиномиальным по импульсам первым интегралом $F$ произвольной степени $N$ c аналитическими коэффициентами на $\frac{N+1}{2}$ или 
$\frac{N+2}{2}($ в зависимости от четности $N)$ попарно различных уровнях энергии $\{H=$ $\left.C_{1}^{2}\right\},\left\{H=C_{2}\right\}, \ldots$. Здесь $C_{j}>0$ для любого $j$. Тогда магнитное поле и метрика зависят лишь от одной переменной и, следовательно, существует линейный по импульсам первый интеграл потока (1.2) на всех уровнях энергии.

Оставшаяся часть раздела посвящена распространению ранее полученных результатов на случай рациональных первых интегралов геодезических потоков в магнитном поле. Во-первых, обобщим утверждение леммы 2 следующим образом.

Лемма 3. Предположим, что магнитный геодезический поток (1.2) на двумерном торе обладает рациональным по импульсам первым интегралом $F=\frac{Q_{N}}{R_{M}}$ на $\frac{N+M+1}{2}$ или $\frac{N+M+2}{2}$ (в зависимости от четности $\left.N+M\right)$ попарно различных уровнях энергии $\left\{H=C_{1}\right\},\left\{H=C_{2}\right\}, \ldots$. Здесь $Q_{N}, R_{M}-$ неоднородные полиномы по импульсам степеней $N$ и $M$ соответственно, $C_{j}>0$ для любого $j$. Тогда $F$ является первым интегралом потока (1.2) одновременно на всех уровнях энергии.

Доказательство леммы 3 полностью аналогично доказательству леммы 2, поэтому не будем его приводить.

Отметим следующий простой технический факт: наличие магнитного поля не дает вклада в уравнения, эквивалентные обращению в нуль коэффициентов скобки Пуассона при мономах старших степеней. Это позволяет автоматически распространить (с минимальными изменениями) результаты, полученные в разд. 2 , на случай магнитного геодезического потока.

Лемма 4. Предположим, что магнитный геодезический поток (1.2) метрики $d s^{2}=\Lambda(x, y)\left(d x^{2}+d y^{2}\right)$ на двумерной поверхности обладает рациональным по импульсам первым интегралом

$$
F=\frac{\sum_{k=0}^{M} \sum_{l=0}^{k} a_{k, l}(x, y) p_{1}^{k-l} p_{2}^{l}}{\sum_{s=0}^{N} \sum_{r=0}^{s} b_{s, r}(x, y) p_{1}^{s-r} p_{2}^{r}}
$$

на одном, нескольких или на всех уровнях энергии, причем коэффициенты $a_{M, l}(x, y), b_{N, r}(x, y)$ при мономах старших степеней числителя и знаменателя - аналитические функции. Положим

$$
\begin{gathered}
f_{1}=a_{M, 0}-a_{M, 2}+a_{M, 4-} \ldots, \quad g_{1}=a_{M, 1}-a_{M, 3}+a_{M, 5}-\ldots, \\
f_{2}=b_{N, 0}-b_{N, 2}+b_{N, 4}-\ldots, \quad g_{2}=b_{N, 1}-b_{N, 3}+b_{N, 5}-\ldots,
\end{gathered}
$$

здесь $a_{M, k}=0$ при $k>M, b_{N, s}=0$ при $s>N$. Тогда имеет место соотношение

$$
\left(\frac{f_{1}-i g_{1}}{f_{2}-i g_{2}}\right)_{x}-i\left(\frac{f_{1}-i g_{1}}{f_{2}-i g_{2}}\right)_{y}=0
$$

Теорема 3. Предположим, что геодезический поток (1.2) метрики $d s^{2}=$ $\Lambda(x, y)\left(d x^{2}+d y^{2}\right)$ на двумерном торе в ненулевом магнитном поле обладает рациональным по импульсам первым интегралом

$$
F=\frac{a_{0}(x, y) p_{1}+a_{1}(x, y) p_{2}+g(x, y)}{b_{0}(x, y) p_{1}+b_{1}(x, y) p_{2}+h(x, y)}
$$


по крайней мере на двух различных уровнях энергии. Предположим, что все коэффициенты $a_{k}(x, y), b_{s}(x, y)$ - аналитические функции, периодические по обоим аргументам, причем хотя бы один из этих коэффициентов нигде не обращается в нуль. Тогда существует линейный по импульсам первый интеграл на всех уровнях энергии.

\section{ЛИТЕРАТУРА}

1. Козлов В. В. Топологические препятствия к интегрируемости натуральных механических систем // Докл. АН СССР. 1979. Т. 249, № 6. С. 1299-1302.

2. Козлов В. В. Симметрии, топология и резонансы в гамильтоновой механике. Ижевск: Изд-во Удмурт. гос. ун-та, 1995.

3. Колокольцов В. Н. Геодезические потоки на двумерных многообразиях с дополнительным полиномиальным по скоростям первым интегралом // Изв. АН СССР. Сер. мат. 1982. Т. 46, № 5. C. 994-1010.

4. Болсинов А. В., Козлов В. В., Фоменко А. Т. Принцип Мопертюи и геодезические потоки на сфере, возникающие из интегрируемых случаев динамики твердого тела // Успехи мат. наук. 1995. Т. 50, № 3. С. 3-32.

5. Денисова Н. В., Козлов В. В. Полиномиальные интегралы геодезических потоков на двумерном торе // Мат. сб. 1994. Т. 185, № 12. С. 49-64.

6. Козлов В. В., Трещев Д. В. Об интегрируемости гамильтоновых систем с торическим пространством положений // Мат. сб. 1988. Т. 135, № 1. С. 119-138.

7. Bialy M. L., Mironov A. E. Rich quasi-linear system for integrable geodesic flow on 2-torus // Disc. Cont. Dynam. Systems. A. 2011. V. 29, N 1. P. 81-90.

8. Bialy M. L., Mironov A. E. Integrable geodesic flows on 2-torus: formal solutions and variational principle // J. Geom. Phys. 2015. V. 87. P. 39-47.

9. Bialy M. L., Mironov A. E. Cubic and quartic integrals for geodesic flow on 2-torus via a system of the hydrodynamic type // Nonlinearity. 2011. V. 24, N 12. P. 3541-3554.

10. Багдерина Ю. Ю. Рациональные интегралы второй степени двумерных уравнений геодезических // Сиб. электрон. мат. изв. 2017. Т. 14. С. 33-40.

11. Collinson C. D. A note on the integrability conditions for the existence of rational first integrals of the geodesic equations in a Riemannian space // Gen. Relativity Gravitation. 1986. V. 18, N 2. P. 207-214.

12. Collinson C. D., O'Donnell P. J. A class of empty spacetimes admitting a rational first integral of the geodesic equation // Gen. Relativity Gravitation. 1992. V. 24, N 4. P. 451-455.

13. Combot Th. Rational integrability of trigonometric polynomial potentials on the flat torus // Regul. Chaotic Dyn. 2017. V. 22, N 4. P. 386-397.

14. Heilbronn G. Integration des equations differentielles ordinaires par la methode de Drach. Paris: Gauthier-Villars, 1956.

15. Pavlov M. V., Tsarev S. P. Classical mechanical systems with one-and-a-half degrees of freedom and Vlasov kinetic equation // Amer. Math. Soc. Transl. 2014. V. 234. P. 337-371.

16. Maciejewski A. J., Przybylska M. Darboux polynomials and first integrals of natural polynomial Hamiltonian systems // Phys. Lett. A. 2004. V. 326, N 3-4. P. 219-226.

17. Агапов C. В. Рациональные интегралы натуральной механической системы на двумерном торе // Сиб. мат. журн. 2020. Т. 61, № 2. С. 255-265.

18. Козлов В. В. О рациональных интегралах геодезических потоков // Нелиная динамика. 2014. T. 10 , № 4. C. 439-445.

19. Aoki A., Houri T., Tomoda K. Rational first integrals of geodesic equations and generalised hidden symmetries // Classical Quantum Gravity. 2016. V. 33, N 19. 195003, 12 pp.

20. Hietarinta $J$. New integrable Hamiltonians with transcendental invariants // Phys. Rev. Lett. 1984. V. 52, N 1057. P. 1057-1060.

21. Переломов А. М. Интегрируемые системы классической механики и алгебры Ли. М.: Наука, 1990.

22. Тен В. В. Полиномиальные первые интегралы систем с гироскопическими силами // Мат. заметки. 2000. Т. 68, № 1. С. 151-253.

23. Тайманов И. А. О первых интегралах геодезических потоков на двумерном торе // Тр. МИАН. 2016. Т. 295. С. 241-260.

24. Agapov S., Valyuzhenich A. Polynomial integrals of magnetic geodesic flows on the 2-torus on several energy levels // Disc. Cont. Dynam. Systems, A. 2019. V. 39, N 11. P. 6565-6583. 
25. Dorizzi B., Grammaticos B., Ramani A., Winternitz P. Integrable Hamiltonian systems with velocity-dependent potentials // J. Math. Phys. 1985. V. 26, N 12. P. 3070-3079.

26. Agapov S. V., Bialy M., Mironov A. E. Integrable magnetic geodesic flows on 2-torus: new examples via quasi-linear system of PDEs // Comm. Math. Phys. 2017. V. 351, N 3. P. 993-1007.

27. Bialy M. L. Rigidity for periodic magnetic fields // Ergod. Theor. Dyn. Syst. 2000. V. 20, N 6. P. 1619-1626.

28. Bialy M. L., Mironov A. E. New semi-Hamiltonian hierarchy related to integrable magnetic flows on surfaces // Cent. Eur. J. Math. 2012. V. 10, N 5. P. 1596-1604.

29. Болотин C. В. О первых интегралах систем с гироскопическими силами // Вестн. Моск. ун-та. Сер. 1. Математика, механика. 1984. Т. 6. С. 75-82.

30. Bolsinov A. V., Jovanovic B. Magnetic geodesic flows on coadjoint orbits // J. Phys. Math. A. 2006. V. 39. P. L247-L252.

31. Burns K., Matveev V.S. On the rigidity of magnetic systems with the same magnetic geodesics // Proc. Amer. Math. Soc. 2006. V. 134. P. 427-434.

32. Ефимов Д. И. Магнитный геодезический поток в однородном поле на комплексном проективном пространстве // Сиб. мат. журн. 2004. Т. 45, № 3. С. 566-576.

33. Ефимов Д. И. Магнитный геодезический поток на однородном симплектическом многообразии // Сиб. мат. журн. 2005. Т. 46, № 1. С. 106-118.

34. Taimanov I. A. On an integrable magnetic geodesic flow on the two-torus // Regul. Chaotic Dyn. 2015. V. 20, N 6. P. 667-678.

35. Царев C. П. Геометрия гамильтоновых систем гидродинамического типа. Обобщенный метод годографа // Изв. АН СССР. Сер. мат. 1990. Т. 54, № 5. С. 1048-1068.

36. Рождественский Б. Л., Яненко Н. Н. Системы квазилинейных уравнений. М.: Наука, 1968.

37. Хайруллин Р. С. К теории уравнения Эйлера - Пуассона - Дарбу // Изв. вузов. Математика. 1993. № 11. С. 69-76.

Поступила в редакиию 10 января 2020 2.

После доработки 2 июня 2020 г.

Принята к публикации 17 июня 2020 г.

Агапов Сергей Вадимович

Институт математики им. С. Л. Соболева СО РАН,

пр. Академика Коптюга, 4, Новосибирск 630090;

Новосибирский государственный университет,

ул. Пирогова, 1, Новосибирск 630090

agapov.sergey.v@gmail.com, agapov@math.nsc.ru 